\title{
Criminologie
}

\section{Notes introductives à l'étude de la criminalité des affaires}

\section{José Rico}

Volume 10, numéro 1, 1977

La criminalité des affaires au Québec

URI : https://id.erudit.org/iderudit/017063ar

DOI : https://doi.org/10.7202/017063ar

Aller au sommaire du numéro

Éditeur(s)

Les Presses de l'Université de Montréal

ISSN

0316-0041 (imprimé)

1492-1367 (numérique)

Découvrir la revue

Citer cet article

Rico, J. (1977). Notes introductives à l'étude de la criminalité des affaires.

Criminologie, 10(1), 8-28. https://doi.org/10.7202/017063ar d'utilisation que vous pouvez consulter en ligne.

https://apropos.erudit.org/fr/usagers/politique-dutilisation/ 


\section{NOTES INTRODUCTIVES \\ A L'ÉTUDE DE LA CRIMINA- LITÉ D'AFFAIRES}

José Rico

\section{PROBLEMATIQUE}

A côté du crime traditionnel, généralement considéré comme un phénomène d'inadaptation sociale propre à des individus tarés, peu instruits, ayant des difficultés matérielles, il existe une autre sorte de délits que l'on classe depuis quelque temps sous des étiquettes diverses (crime en col blanc, crime d'affaires), bien qu'ils désignent finalement une réalité unique, caractérisée par des éléments communs.

On connaissait, surtout à partir des travaux de Sutherland, l'existence de la criminalité en col blanc, basée sur les idées d'organisation et de profits considérables, et commise par les membres les plus aisés et les plus réputés de la société, particulièrement en relation avec leur activité professionnelle. $\mathrm{Ce}$ qui caractérise le plus les auteurs de ces délits est leur tendance à exploiter le pouvoir de l'influence qu'on leur a confié pour la satisfaction d'intérêts personnels; les manières ingénieuses qu'ils emploient dans la commission de leurs actes, qui excluent presque totalement toute possibilité d'échec ou de découverte ; finalement, leur attitude devant les actes commis, considérés comme de simples illégalités et non pas comme des crimes. On constate en outre une absence presque complète de réprobation sociale véritable s'attachant à la condamnation pénale d'un homme d'affaires.

Le problème de la criminalité d'affaires n'est certes pas neuf, mais il se pose avec acuité dans une société qu'anime le désir de croissance et que les règles morales n'arrêtent plus. Il s'agit d'un domaine mal exploré, où les seuls faits connus semblent être un chiffre noir considérable et d'énormes bénéfices obtenus illégalement. Par ailleurs, une des principales difficultés pour l'étude de cette forme de criminalité consiste dans le caractère secret ou, tout au moins, peu accessible des sources de renseignement. Cependant, cette étude s'impose, en particulier si l'on veut que la criminologie puisse jouer un rôle essentiel d'auxiliaire de la justice et se mettre plus efficacement au service d'une société plus juste, en se consacrant principalement à l'analyse de certaines 
formes de criminalité dont les conséquences politico-économiques pourraient, selon l'expression du sénateur américain Kefauver, faire perdre aux citoyens le pouvoir d'orienter leur propre progrès économique et, à la limite, leur liberté de vivre, de parler, d'agir et de se défendre ${ }^{1}$.

La plupart des Etats occidentaux présentent aujourd'hui, en dépit de leurs structures sociales et économiques très différentes, une criminalité économique identique. Celle-ci dépend néanmoins de la tendance fondamentale des systèmes économiques. L'économie occidentale, organisée selon le principe de la libre concurrence et basée sur la réalisation de profits et sur la croissance, incite l'homme d'affaires à dépasser une légitime marge de bénéfices pour aboutir à une politique de profit à tout prix. Cette soif de profit pousse certaines personnes à dépasser les bornes et les conduit ou les induit à des actes criminels. La découverte et l'évaluation de tels comportements criminels se compliquent aussi en raison des transformations que le processus économique a subies depuis le $\mathrm{XIX}^{e}$ siècle. En raison du caractère complexe et anonyme de l'économie moderne, ainsi que de l'expansion de nouveaux modes de payement au comptant et d'autres procédés simples et des empiétements interventionnistes de la politique économique sur le processus économique dit naturel, de nouvelles formes de criminalité sont apparues, rendant toujours plus difficiles leurs qualifications juridique et économique. Cette insécurité est particulièrement prononcée dans un État qui respecte en principe la liberté de concurrence et qui n'a érigé qu'un vaste cadre de très vagues normes juridiques en cette matière ${ }^{2}$.

\section{2. ÉTENDUE ET CONSÉQUENCES}

Les délits contre la libre concurrence occupent, dans les pays occidentaux, le premier rang en ce qui concerne cette forme de criminalité (accords cartellaires et concurrence déloyale, fraudes dans le domaine des subventions, escroqueries sur l'épargne à long terme, primes, avantages et rabais illicites, corruption des

1. Cité par G. Kellens, \& Le crime en col blanc : sa place dans une criminologie économique ', Revue de science criminelle, no 4, 1974, p. 807 821.

2. K. Tiedemann, * La criminalité socio-économique : aspects internationaux et le droit comparé $*$, Revue de science criminelle, $\mathrm{n}^{\circ} 4,1974$, p. 749-763 et a La criminalité d'affaires dans l'économie moderne *, Revue internationale de criminologie et de police technique, $\mathrm{n}^{\circ} 2,1975$, p. 147-158. 
employés d'entreprises commerciales, violation de secrets, espionnage industriel). Les systèmes économiques dirigistes connaissent une criminalité des fonctionnaires qui se caractérise par la corruption, l'avilissement des prix, l'établissement de faux certificats et un considérable marché noir. Dans les systèmes à économie socialiste, le «délit de fonction» peut, à certains égards, être rapproché du crime en col blanc du supercapitalisme nordaméricain. À cela s'ajoutent les délits « neutres 》, qui ne dépendent pas d'un système particulier et qui sont, parmi de nombreux autres, des escroqueries dans la circulation des biens et des services, telles que les factures surfaites, les livraisons de moindre valeur, les détournements et les abus de confiance, les dommages à la propriété par destruction des moyens de production, etc. Sans oublier la criminalité dite des ordinateurs électroniques, qui constitue une des conséquences prévisibles d'un développement trop rapide des nouvelles méthodes techniques : la manipulation des données et des programmes, leur destruction, l'emploi non autorisé de l'installation, l'espionnage, etc., sont autant d'aspects de cette nouvelle criminalité qui cause des dommages énormes et reste très difficile à découvrir ${ }^{3}$.

Estimer l'importance de la criminalité économique ne peut se faire qu'à l'aide de vagues conjectures ou d'extrapolations plus ou moins convaincantes. Les recherches sur cette catégorie de criminalité sont peu nombreuses et les rares données disponibles n'ont pas été compilées de façon systématique.

Selon la Commission Katzenbach, les pertes occasionnées annuellement par la fraude fiscale aux Etats-Unis oscillaient entre 25 et 40 milliards de dollars et celles découlant des ventes illégales de drogues et de produits pharmaceutiques ou des évaluations frauduleuses dans les réparations des maisons dépassaient chacune le demi-milliard de dollars ${ }^{4}$. Au Canada, les 329 spécialistes de la police fédérale qui composent la section des crimes commerciaux, travaillaient en 1975 sur environ 2600 cas ayant causé, cette année, des pertes évaluées à plus de 200 millions de dollars $^{5}$; les recherches effectuées par la Commission Prévost

3. K. Tiedmann (1974), note 2, p. 755.

4. The President's Commission on Law Enforcement and Administration of Justice, Task Force Report (1967), Crime and its Impact : An Assessment, Washington (D.C.), U.S. Government Printing Office, p. 103104.

5. La Presse, 24 septembre 1975. 
ont, par ailleurs, montré que 1225 cas de faillites survenues au Québec en 1965, dont environ la moitié était des faillites frauduleuses, ont entraîné des pertes matérielles de quelque 77 millions ${ }^{6}$.

En France, selon les déclarations d'un porte-parole du ministère des Finances au représentant de la revue l'Express, la proportion de la fraude fiscale serait, pour la moyenne de 1960 à 1964 , de 10 à $15 \%$ du total des rentrées fiscales, soit de 15 à 23 milliards de francs actuels ${ }^{7}$; des estimations plus récentes établissent le montant de ces pertes à plus de 50 milliards de francs nouveaux, c'est-à-dire entre 20 et $25 \%$ du budget du pays ou l'équivalent des crédits de l'Éducation nationale ${ }^{8}$.

En Belgique, la fraude fiscale jouit d'un véritable droit de cité et bénéficie des préjugés favorables accordés au « fait acquis 》. Même si son importance réelle est sujette à contestation et difficile à préciser, les chiffres cités au terme d'études sérieuses menées par le professeur Frank ne laissent aucun doute quant à son ampleur et à son caractère généralisé. Ainsi, en 1953, on estimait que dans le seul domaine des revenus professionnels des particuliers, 6 à 7 milliards de francs belges échappaient à l'impôt direct, en 1965, l'ensemble de la fraude fiscale était estimée à 20 milliards et les dernières études avancent même le chiffre de 50 milliards ${ }^{\circ}$.

Le gouvernement de la République fédérale d'Allemagne estime les dommages matériels annuels résultant de la criminalité économique à 15 millions de DM. Cette évaluation ne paraît guère exagérée, compte tenu du grand pourcentage de cas non élucidés dans diverses branches économiques. Par ailleurs, 250000 effets de change, représentant 500 à 680 millions de $\mathrm{DM}$, sont protestés chaque année et plus d'un million de chèques dépassant le milliard de DM restent chaque année impayés. Le pourcentage d'actes criminels dans ces chiffres est difficilement estimable, étant

6. La Société face au crime, vol. 3, t. 2 : le Crime au Québec : les sommets de la criminalité québécoise, Editeur officiel du Québec, 1969, p. 29-32. p. 141-142.

7. J. Cosson (1971), les Industriels de la fraude fiscale, Paris, Seuil,

8. Ch. De Brie et P. Charpentier (1975), F... comme fraude fiscale, Paris, Alain Moreau, p. 9.

9. R. Henrion (1974) * Le droit fiscal et les pénalités ", dans les Frontières de la répression, vol. 1, Bruxelles, éd. de l'Université de Bruxelles, p. 171-187. 
donné que les banques, qui ont peu d'espoir de voir aboutir une plainte pénale, n'entendent pas perdre de temps, désirent sauvegarder le secret bancaire et renoncent généralement à porter plainte ${ }^{10}$.

La criminalité économique a des conséquences encore plus graves que ces importants dommages matériels. Les violations des dispositions légales concernant les aliments, les drogues et la protection de l'environnement, les infractions commises par les médecins dans l'exercice de leur profession sont susceptibles de causer des dommages irréparables à la santé des particuliers. Sont également à craindre ses répercussions immatérielles sur l'ordre économique, sur le comportement général des hommes d'affaires, sur le climat moral de la société. Certains auteurs parlent de « l'effet contagieux des délits économiques » (des branches entières imitent le modèle que représente le malfaiteur isole), ainsi que de leur «effet en spirale » (les infractions de cette nature sont souvent commises à l'aide de délits caractérisés : falsification de documents, émission de fausses pièces justificatives, corruption, etc.). La criminalité économique, qui conduit à une distorsion de la concurrence, occasionne l'effondrement des petites entreprises et le congédiement des travailleurs, détruit la confiance que les groupements économiques pouvaient avoir dans l'intégrité de la vie économique et cause finalement de sérieux dommages aux institutions sociales et économiques d'un pays.

Il convient en outre de souligner la croissante internationalisation de cette forme de criminalité qui tire parti du système économique tel qu'il est actuellement organisé. Depuis longtemps, les frontières nationales sont largement dépassées dans le domaine du crédit, des subventions, des payements, du financement et du placement de capitaux, de l'évasion fiscale, de l'aide aux pays en voie de développement. Les grandes entreprises de tous les pays du monde connaissent parfaitement la liste des nombreux «paradis fiscaux $\gg$ et certains organismes supranationaux, telle la Communauté européenne, sont tombés, depuis leur naissance, sous la coupe de véritables bandes d'escrocs ${ }^{11}$.

Les pays en voie de développement sont particulièrement exposés à ces formes de criminalité affectant l'économie nationale.

10. K. Tiedemann (1975), note 2, p. 149-150.

11. Sur les escroqueries au sein de la Communauté européenne, voir Tiedemann, ibid., p. 150-152. 
Dans la plupart de ces nations, les conséquences sociales et économiques de la criminalité économique sont infiniment plus graves que celles découlant des formes classiques de criminalité violente ou contre la propriété ${ }^{12}$.

Malgré l'importance de ce phénomène, les recherches sur la criminalité d'affaires, extrêmement rares par ailleurs, dépassent à peine les analyses juridiques ou criminalistiques.

\section{REVUE DE LA LITTÉRATURE}

C'est Edwin C. Hill qui, le premier, attira l'attention, devant le Congrès international sur la prévention et la répression du crime (Londres, 1872), sur l'importance grandissante de la criminalité dans le domaine des affaires; il offrait comme exemple certains agissements illégaux d'agents d'immeubles, de manufacturiers et d'autres personnes « honnêtes ».

Cependant, l'idée de l'étude de la criminalité en fonction de l'origine sociale des auteurs a été développée avec force, pour la première fois, par Gabriel Tarde ${ }^{13}$. Partant de sa théorie de l'imitation, Tarde posa le problème des rapports de la criminalité et de la profession et nota l'existence d'une délinquance professionnelle liée à la profession exercée. Pour lui, la criminalité pouvait se manifester à l'occasion d'une profession ordinaire et constituer une profession organisée.

En 1907, le sociologue Edward A. Ross décrivait les principaux traits de certaines personnes de la haute société, qui, même quand elles commettaient des crimes, n'attiraient aucune réaction sociale ${ }^{14}$. En 1935, A. Morris, reprenant le thème, parlait des "criminels de la haute société », qui n'ont jamais été qualifiés ou présentés comme un groupe particulier et assez concret pour attirer la désapprobation du public. Ces personnes n'ont

12. Ve Congrès des Nations unies pour la prévention du crime et le traitement des délinquants, Genève (1975). Formes et dimensions nouvelles - nationales et transnationales - de la criminalité, document de travail préparé par le Secrétariat, p. 7-17; voir également A. Sampson (1973), ITT. l'Etat souverain, Paris, Alain Moreau.

13. G. Tarde (1896), La criminalité professionnelle », Archives d'anthropologie criminelle, p. 538-560, cité par J. Pinatel, \& La criminalité dans les différents cercles sociaux $*$, Revue de science criminelle, no 3 , 1970 , p. 677-685.

14. E.A. Ross, «The Criminaloid *, dans The Atlantic Monthly, 99 (1907), p. 44-50, repris dans G. Geis édit. (1968), White Collar Criminal. Thirty two Papers, New York, Atherton Press, p. 25-33. 
jamais été conduites en masse en prison, ni enfermées en commun dans des lieux aptes à l'étude et à la vérification de leurs particularités, afin qu'on puisse parler d'un type humain spécifique ; au contraire, elles se mêlent à la foule et vivent leur vie comme des amis ou membres actifs de clubs et de sociétés religieuses; quelquefois même, elles contribuent aux organisations pour la lutte contre la délinquance juvénile et aident le pouvoir législatif à voter des lois contre le crime; la seule différence entre ces individus et les personnes honnêtes appartenant à leur classe sociale réside dans une moindre sensibilité éthique sur certains points, qui serait surtout due aux relations serrées qu'ils entretiennent avec le modèle criminel qui est le leur ${ }^{15}$.

Mais c'est surtout Sutherland qui a établi les bases d'une théorie de la criminalité en col blanc. Dans son allocution présidentielle devant la Société américaine de sociologie (1939), il montra, documents à l'appui, les importantes infractions dont se rendaient coupables les sociétés en matière de prix, de relations de travail, de droits d'auteur, de produits alimentaires et pharmaceutiques. Son étude portait sur le casier judiciaire de 70 grandes sociétés et de leurs filiales, d'une vie de quelque 45 ans en moyenne, choisies sur deux listes des 200 plus grandes entreprises non financières des États-Unis. En fait, $16 \%$ seulement des 980 décisions prononcées contre ces sociétés émanaient des juridictions pénales, mais plusieurs sociétés avaient encouru quatre condamnations pénales et pouvaient être considérées comme des criminels d'habitude. Sans doute, selon Sutherland, les infractions économiques ne donnent pas lieu au processus classique " policetribunal-prison », mais plutôt à une comparution devant des commissions administratives ou des juridictions civiles. Cependant et c'est la thèse de l'ouvrage - la criminalité des classes supérieures ne se distingue essentiellement de la criminalité dite ordinaire que par la procédure et il existe bel et bien une criminalité des classes aisées. Ainsi, pour Sutherland, la criminalité en col blanc désigne les activités illégales déployées par des personnes respectables et de classe sociale élevée en relation avec leurs activités professionnelles ${ }^{16}$. 158.

15. A. Morris (1935), Criminology, New York, Longmans, p. 157.

16. E.H. Sutherland, - White Collar Criminality , American Sociological Review, 5 (1940), p. 1-12. Cet auteur reprit le même thème dans les publications suivantes : - Crime and Business \$, The Annals of the American Academy of Political and Social Science, 217 (1941), p. 112-118; - Is 
Depuis Sutherland, la bibliographie sur la criminalité en col blanc n'a cessé de se développer. Des progrès considérables ont été réalisés aussi bien dans la compilation des données que dans la clarification des concepts théoriques. La plupart des études ont été faites par des auteurs américains. Des données factuelles ont été présentées par Clinard, Hartung, Newman et, en particulier, par le rapport des audiences du Sénat américain sur les infractions commises par 29 compagnies d'équipement et d'accessoires électriques dont la General Electric et la Westinghouse en $1961^{17}$, alors que des éclaircissements théoriques ont été effectués par Cressey, Geis, Bloch et Geis, Newman, Clinard, Tappan, Quinney et autres ${ }^{18}$. Souvent, les ouvrages les plus stimulants pour la recherche sont le fait de journalistes ${ }^{19}$. Le résultat presque inévitable de ce quasi-monopole a été que tous ces écrits, ainsi que les discussions théoriques qui en découlent, se réfèrent essentiellement aux conditions nord-américaines : législation antitrust, violations des normes relatives aux grosses affaires, etc. ${ }^{20}$

Il convient de mentionner que ni cette forme de criminalité ni l'importante recherche de Sutherland ne firent à l'époque l'objet d'une large publicité dans les media d'information; une grande partie des documents originaux furent enterrés dans les dossiers des organismes chargés de coordonner les recherches et quand

- White Collar Crime " Crime? ", American Sociological Review, 10 (1945), p. 132-139; et White Collar Crime, New York, The Dryden Press, 1949 (réédition 1961, New York, Holt, Rinehart et Winston, avec préface de D.R. Cressey).

17. M.B. Clinard (1952), The Black Market, New York, Rinehart et - Criminological Theories of Violations of Wartime Regulations *, Am. Soc. Rev. 11 (1946), p. 258-270 ; F.E. Hartung, * White Collar Offenses in the wholesale Meat Industry in Detroit », American Journal of Sociology, 56 (1950), p. 25-35; D.J. Newman, - White Collar Crime», Law and Contemporary Problems, 23 (1958), p. 735-753; R.A. Smith, * The Incredible Electrical Conspiracy ", dans M.E. Wolfgang et al. (1962), Sociology of Crime and Delinquency, New York, Wiley, p. 357-372.

18. D.R. Cressey, "The Criminal Violation of Financial Trust *, Am. Soc. Rev. 15 (1950), p. 738-743; G. Geis, c Toward a Delineation of White Collar Offenses *, Sociological Inquiry, 32 (1962), p. 160-171; H.A. Bloch et G. Geis (1962), Man Crime and Society, New York, Random House ; P.W. Tappan, e Who is the Criminal ? *, Am. Soc. Rev., 12 (1947), p. 96-103; R. Quinney, Occupational Structure and Criminal Behaviour : Prescription Violations by Retail Pharmacist s, Social Problems, 11 (1963), p. 179-185; J.E. Carlin (1966), Lawyer's Ethics, New York, Roussel Sage Foundation ; J.C. Spencer (1966), - White Collar Crime ", dans T. Grygier et al. édit. (1965), Criminology in Transition, Londres, Tavistok, p. 233-266. Laffont.

19. Ch. Raw et al. (1972), Voulez-vous vraiment être riche?, Paris,

20. H. Mannheim (1965), Comparative Criminology, vol. 1, Londres, Routledge and Paul Kegan, ch. 21, p. 469-498. 
finalement ils furent classés et interprétés, ils furent publiés uniquement dans des périodiques savants destinés à des universitaires, le public n'ayant jamais été informé.

En Europe, les premières recherches sur la criminalité d'affaires ont été réalisées vers les années 50. En France, elles portaient sur l'escroquerie et l'émission de chèques sans provision $^{21}$ ainsi que sur l'abus de confiance ${ }^{22}$; dans ce pays, une attention spéciale a été consentie à la fraude fiscale, qui a fait l'objet, depuis 1971, de plusieurs études importantes ${ }^{23}$. En Belgique, Kellens a effectué des recherches sur les ventes à tempérament et sur la banqueroute ${ }^{24}$, et en Allemagne, Tiedemann a étudié la criminalité d'affaires dans l'économie moderne, notamment son incidence au sein de la Communauté européenne ${ }^{25}$. Par ailleurs, à la suite d'une réunion des administrateurs douaniers européens, célébrée en 1974 à Bruxelles, au cours de laquelle on dénonça l'accroissement des tentatives de fraude liées à la complication des dispositions communautaires, qui ne permet d'en garantir ni un contrôle efficace ni la correcte application, une recherche a été entreprise sur la délinquance d'affaires dans le cadre des activités de cette Communauté ${ }^{26}$.

Mis à part quelques données de base obtenues à l'occasion des activités de diverses commissions d'enquête ${ }^{27}$, les recherches sur la criminalité d'affaires sont inexistantes au Canada.

21. A. Davidovitch, * L'escroquerie et l'émission de chèques sans provision ", l'Année sociologique, 1955-1956, p. 3-130.

22. H. Levy-Bruhl, \&Enquête statistique sur l'abus de confiance *, dans Actes du II ${ }^{\mathbf{e}}$ Congrès international de criminologie, Paris, P.U.F., vol. IV, p. 35-41.

23. J. Cosson, note 7 ; M. Denuzière (1973), Enquête sur la fraude fiscale, Paris, J.-C. Lattès, éd. spéciale; Ch. de Brie et P. Charpentier, op. cit., note 8 et (1973), l'inégalité par l'impôt, Paris, Seuil. Pour une description essentiellement juridique de cette forme de criminalité, voir Mireille Delmas-Marty (1973), Droit pénal des affaires, Paris, P.U.F.

24. G. Kellens, Aspects criminologiques des ventes à tempérament ${ }^{\prime}$, Revue de droit pénal et de criminologie, $\mathrm{n}^{\circ} 8$, mai 1967, p. 779-845 et (1974), Banqueroute et banqueroutiers, Bruxelles, Dessart et Mardaga (ce livre contient un aperçu des recherches effectuées dans ce secteur de la criminalité).

25. Voir les articles déjà cités en note 2 .

26. La délinquance d'affaires dans le cadre des règles communautaires $»$, recherche exploratoire, rapport final élaboré, sous la direction de Mireille Delmas-Marty, par Béatrice Arnaud et al. (1975), Université de Lille II, Institut de criminologie et des sciences criminelles.

27. La Commission Prévost a étudié le dossier des faillites frauduleuses au Québec (voir référence, note 6 ); plus récemment, la Commission d'enquête sur le crime organisé a montré, également au Québec, le rôle joué par le crime organisé dans cette forme de criminalité. 


\section{PERSPECTIVES DE RECHERCHE}

L'étude scientifique de la criminalité d'affaires présente des difficultés considérables. Il s'agit, en premier lieu, d'un domaine où le chiffre noir semble extrêmement élevé, les auteurs n'étant que très rarement pris sur le fait. C'est également un sujet qui, en raison de ses prolongements politiques, peut facilement aboutir à des conclusions partisanes et non scientifiques ${ }^{28}$. Il présente, par ailleurs, à cause de ses liens étroits avec les problèmes sociaux contemporains, une ampleur et une profondeur d'une telle importance qu'il risque de mener à des généralisations abusives. Son caractère nouveau empêche finalement les conclusions définitives.

Le chercheur doit cependant essayer de surmonter ces difficultés, d'éviter ces dangers, car cette sorte de criminalité constitue une des formes les plus représentatives des tendances criminelles de notre époque.

Les principales questions que la criminalité d'affaires pose à la recherche ont trait à la définition du concept de criminalité d'affaires, à l'étude de l'infracteur et à l'analyse de la réaction sociale.

\section{A. La définition de la criminalité d'affaires}

Sous des appellations familières - criminalité d'affaires en Europe, crime en col blanc en Amérique du Nord - sont regroupées des infractions pénales très diverses qui appartiennent à la criminalité d'affaires lorsqu'elles sont commises dans certaines conditions. La question se pose alors de savoir quelles sont ces conditions, quels critères faut-il retenir pour qu'un crime soit qualifié d'affaires?

La difficulté est de taille. Lesquels, en effet, parmi les délits suivants - fraude fiscale, banqueroute, spéculation illicite des prix, faillite frauduleuse, abus de biens sociaux, pots-de-vin, révélation de secrets, abus de confiance, publicité mensongère, contrefaçon, escroquerie, faux en écriture, surestimation ou sous-estimation d'actifs, pseudosolvabilité, trafic d'influence, manipulation des cotes de la bourse, détournement de fonds, concurrence déloyale, etc. — relèvent de la criminalité d'affaires, lesquels de la criminalité classique contre les biens et lesquels ne sont que

28. Clinard (1946), note 17, p. 258. 
l'utilisation intelligente des possibilités légales de pratiquer les affaires?

Ainsi, les expressions «criminalité d'affaires » ou « criminalité en col blanc » sont davantage descriptives qu'opérationnelles. Mais quelles sont la signification et la portée de ces termes?

Pour Sutherland, le crime en col blanc était le crime commis par une personne respectable et possédant un statut social élevé au cours de ses activités professionnelles. Trois éléments sont donc essentiels dans cette définition : 1) il s'agit, en premier lieu, d'un crime ; 2) c'est en deuxième lieu, un acte commis par une personne respectable et de niveau socio-économique élevé ; 3) l'acte est finalement commis dans le cadre de ses activités professionnelles.

1) Le crime en col blanc est-il véritablement un crime ? Il s'agit d'une question très controversée. D'après Sutherland, le crime en col blanc réunit les critères essentiels pour qu'un acte constitue une infraction : comportement interdit et puni par l'État, en raison de son caractère dommageable pour le bien public. Caldwell et Tappan sont cependant d'avis que personne ne doit être considéré comme criminel tant qu'il n'a pas été déclaré coupable, à la suite d'un procès pénal, d'une infraction à la loi pénale; les violations des dispositions administratives ne doivent pas, par contre, être retenues ${ }^{29}$.

Toujours selon Sutherland, le crime en col blanc se distingue des autres crimes par la procédure appliquée. Les procédures pénales sont généralement employées lorsqu'il s'agit de délits ordinaires ; par contre, les personnes accusées d'un crime en col blanc sont rarement arrêtées et ne sont pas traduites devant les cours criminelles ou condamnées à la prison mais plutôt convoquées devant des commissions administratives ou des juridictions civiles, et lorsque des décisions sont prises contre elles, il s'agit généralement d'injonctions, de mises en demeure ou d'ordres les invitant à ne pas persister dans leur conduite. Ces différences s'expliquent par le statut socio-économique élevé des délinquants en col blanc.

2) Les crimes en col blanc sont commis exclusivement par des personnes de niveau socio-économique élevé. Les actes de

29. R.G. Caldwell, \& A Reexamination of the Concept of White Collar Criminal », Federal Probation, 22 (1958) p. 30-36; Tappan, note 18. 
ceux qui, appartenant à un rang socio-économique moins élevé, violent occasionnellement ou systématiquement les règles de leur corporation (avocat véreux, médecin marron, sage-femme avorteuse, etc.) devraient, dans cette perspective, être exclus.

Plusieurs auteurs ont toutefois proposé d'étendre le domaine du crime en col blanc à toute la délinquance professionnelle, sans égard à la classe sociale du délinquant ${ }^{30}$. Certains ont mis l'accent sur la façon dont cette sorte de crimes sont commis ${ }^{81}$; c'est ainsi que Newman voit des crimes en col blanc dans tous les crimes ingénieux, indépendamment du rang socio-économique de leurs auteurs (par exemple, coupage de lait par les paysans, réparation inutile d'une voiture ou d'un appareil de télévision). D'autres finalement pensent qu'au lieu d'étendre la notion plus ou moins acceptée de crime en col blanc, il serait préférable d'établir d'autres notions plus larges, comme par exemple celle de «crime occupationnel », qui comprendrait également le «crime en col bleu », c'est-à-dire les infractions commises par des ouvriers dans l'exercice de leurs activités professionnelles et, notamment, les notes injustifiées de garagistes, d'horlogers ou de réparateurs de radios pour des réparations non effectuées ${ }^{32}$.

Certains criminologues contestent énergiquement cette extension de la notion de crime en col blanc. En effet, souligne Kellens,

si l'on peut admettre éventuellement une extension de la notion de « criminel en col blanc » pour y englober les classes moyennes, à condition qu'on le dise clairement, il importe de ne pas perdre de vue que l'objet essentiel du concept de crime en col blanc est d'attirer l'attention sur des crimes qui auparavant n'étaient pas envisagés en criminologie et que ce n'est que lorsque les conceptions des criminologues, des hommes de loi et du public en général à l'égard des criminels en col blanc au sens classique du terme auront changé, que le concept de crime en col blanc, envisagé par Sutherland lui-même comme provisoire, pourra disparaître. Entre-temps lui enlever tout contenu de classe serait le dénaturer au point de lui faire perdre toute signification ${ }^{33}$.

30. A. Normandeau, * Les * déviations en affaires * et le * crime en col blanc *, Revue internationale de criminologie et de police technigue, $\mathrm{n}^{\circ} 4,1965$, p. $247-258$; Clinard (1946), note 17.

31. Newman (1958), note 17.

32. E.R. Quinney, "The Study of White Collar Crime : Toward a Reorientation in Theory and Research *, Journal of Criminal Law, Criminology and Police Science, 55, (1964), p. 208-214.

33. G. Kellens (1974), note 1, p. 816. 
Si les virtualités de ce concept ont été insuffisamment dégagées ou exploitées, c'est essentiellement parce que la recherche a été négligée dans ce domaine au profit de discussions académiques plus ou moins valables.

3) Finalement, les crimes commis par des personnes de haut rang social ne peuvent être caractérisés comme «crimes en col blanc > que s'ils sont liés à l'activité professionnelle de ces personnes.

Les Allemands ont, par contre, élargi la portée de cette notion en classant, sous le nom de Kavaliersdelikt, tous les crimes commis par des personnes en col blanc grâce à leur condition privilégiée dans la société, même si ces crimes n'ont aucun rapport avec leurs activités professionnelles (par exemple, le duel, le port illicite d'armes ou, dans le domaine sexuel, la séduction et l'adultère). Plus récemment, la doctrine allemande a raffiné cette notion et distingue deux catégories de « délits de chevalier ». Dans une première catégorie, elle range certains comportements frauduleux (fraude fiscale et douanière, vols de matières premières, vols sur les chantiers, vols de «souvenirs » par les touristes, etc.), qui ont pour trait commun une forme d'émulation fondée sur des rationalisations telles que " tout le monde le fait », « ce n'est pas un vol » jointe à un élément sportif lié à un certain goût du risque. Elle classe dans la deuxième catégorie une série d'infractions qui sont généralement commises avec une conscience amoindrie du caractère délictueux de l'acte accompli, due, soit au fait que le délinquant éprouve le sentiment d'user de biens qui appartiennent au domaine public (braconnage, droits d'auteur), soit à la trop grande nouveauté d'une législation pénale, soit au fait qu'une législation ne correspond plus aux mours du temps ${ }^{34}$.

4) A ces éléments essentiels de la définition du crime en col blanc, on a ajouté d'autres caractéristiques susceptibles d'aider à une meilleure compréhension du problème. Pour certains, le crime en col blanc : a) consiste en une exploitation des crédules (les victimes sont, de préférence, choisies parmi la foule et, surtout, parmi les personnes qui ont peu de possibilités d'obtenir des biens vitaux, de contrôler les services proposés ou de réagir); b) est réalisé d'une manière ingénieuse, qui exclut presque sa

34. G. Kellens, Crime en col blanc et stigmatisation s, Revue de droit pénal et de criminologie, $n^{\circ} 3$, décembre 1970, p. 327-338. 
découverte (les criminels en col blanc échappent, en général, à la poursuite pénale et à leur place, ce sont de simples comparses qui sont arrêtés); et c) est commis par des personnes qui sont au courant du caractère illégal de leur conduite mais qui ne croient pas, pour diverses raisons (droit personnel à violer la loi lorsqu'il s'agit de la réalisation d'un projet dans l'intérêt de la société, loi injuste ou irrationnelle, phénomène habituel), qu'il s'agit d'un comportement criminel ${ }^{35}$. D'après les Nations unies qui utilisent, pour se référer à la criminalité économique la notion plus large de " crime en tant qu'entreprise lucrative », celui-ci tend à posséder les caractéristiques suivantes, ou du moins une partie d'entre elles : a) il est perpétré essentiellement dans un but lucratif et met en cause une quelconque forme de commerce, d'industrie ou d'activité professionnelle; b) il implique une certaine forme d'organisation, au sens d'un ensemble ou d'un système de relations plus ou moins établies entre les parties qui commettent les actes criminels ; c) il suppose soit l'usage, soit l'abus des formes et techniques légitimes du commerce, de l'industrie ou des activités professionnelles; d) généralement, mais non nécessairement, les personnes impliquées dans ce genre de criminalité jouissent d'une position sociale relativement élevée ou d'un pouvoir politique, ou les deux ${ }^{36}$.

En France, des efforts ont été consentis en vue de délimiter le champ de la criminalité d'affaires. Pour Mme Delmas-Marty, l'étude du droit pénal des affaires suppose, d'une part, que soient définies les affaires (l'entreprise devrait être la notion de base) et, d'autre part, que soient dêterminées les infractions qui relèvent de lui et le constituent, car, à la limite, de nombreux délits de droit commun (vols commis par les employés d'un magasin, attentat à la pudeur commis par un président-directeur général contre une secrétaire) pourraient être considérés comme appartenant aux affaires. La criminalité d'affaires devrait comprendre, selon cet auteur, certaines infractions de droit commun qui jouent un rôle important dans la vie des affaires, où elles prennent une coloration particulière (par exemple, escroquerie ou abus de confiance) et toutes les infractions spéciales à la vie des affaires ${ }^{37}$.

Ce qui caractériserait surtout cette forme de criminalité serait le fait qu'elle a pour cadre une affaire portant sur des sommes

36. Voir référence, note 12 , p. 7.8 .

37. Mireille Delmas-Marty (1973), note 23, p. 7-9. 
importantes; elle se distingue également par la complexité technique des mécanismes mis sur pied pour la commettre.

\section{B. L'auteur}

Les questions évoquées à l'égard du criminel d'affaires sont simples en apparence : qui ? et pourquoi ${ }^{38}$ ?

1) Qui est ce délinquant d'affaires ? À quoi le reconnaîton? Peut-on le définir avec précision?

Le critère juridique ne saurait être exclusivement retenu. En effet, à côté de certaines infractions (abus de biens sociaux et, en général, les délits de société), qui caractérisent la criminalité d'affaires et pourraient fournir des critères utiles de sélection, il existe d'autres infractions (escroqueries, abus de confiance, recel, fraude fiscale, etc.) qui appartiennent aussi bien à la criminalité d'affaires qu'à la délinquance « ordinaire » contre les biens; la pratique judiciaire montre, par ailleurs, qu'à l'heure actuelle beaucoup de criminels d'affaires sont précisément poursuivis sur la base de ce droit pénal commun.

D'autres critères peuvent cependant être utilisés : le critère objectif, résultant de l'inclusion des faits imputés dans un courant d'affaires ; le critère subjectif, fondé sur le comportement psychologique de l'agent.

Certains cliniciens soutiennent que les motivations du criminel d'affaires et les mobiles du délinquant « ordinaire » contre la propriété sont très différents. Le Dr Fontan, par exemple, affirmait, lors des journées régionales de criminologie, tenues à Lille (France) en octobre 1973, qu'il fallait distinguer entre la conduite "pervertie », qui est celle de l'homme honnête que son inaptitude ou des difficultés accidentelles ont poussé à transgresser la règle, et la conduite " perverse », qui est celle de l'homme d'affaires malhonnête dès le départ, ce qui est beaucoup plus rare ${ }^{39}$. L'acceptation de la première solution aurait l'avantage de reconnaître un domaine plus spécifique à la criminalité d'affaires ; elle correspond, par ailleurs, à la définition du criminel en col blanc qu'évoquent de nombreux auteurs.

38. Mireille Delmas-Marty, \& La criminalité d'affaires ", Revue de science criminelle, $\mathrm{n}^{\circ} 1,1974$, p. 45-55.

39. Voir le compte rendu de ces journées dans la Revue de science criminelle, no 1,1974, p. 197-199. 
En comparant les structures caractérielles du criminel professionnel et du délinquant en col blanc, Pinatel constate que tous deux sont égocentriques, labiles, agressifs et indifférents à un haut degré, mais le criminel en col blanc est un hyperadapté social, tandis que le criminel professionnel, par suite de son incapacité à exercer un métier défini, est un inadapté social ${ }^{40}$; pour l'éminent criminologue français, le criminel d'affaires a une émotivité profonde et dissimulée et possède une volonté de puissance que l'on peut expliquer par un sentiment d'infériorité.

Toujours sur le plan des caractéristiques du délinquant d'affaires, on constate en général que les auteurs principaux sont d'un rang social comparable à celui des dirigeants d'entreprises " orthodoxes », ce qui les différencie des délinquants « ordinaires ». Par contre, les intermédiaires ou hommes de "paille » qui interviennent souvent à l'occasion de ce type de criminalité, sont sociologiquement très proches des délinquants ordinaires, parmi lesquels ils sont souvent recrutés.

2) Si la détermination des raisons qui ont poussé un individu vers la délinquance est une tâche très difficile, cette recherche est particulièrement délicate dans le domaine de la délinquance d'affaires, qui n'est pas toujours ressentie comme une délinquance véritable.

Il convient, à cet égard, d'analyser le comportement de l'auteur, mais aussi d'évoquer le rôle de la victime et de l'opinion publique.

En ce qui concerne l'auteur, deux facteurs, apparemment opposés, semblent jouer un rôle criminogène. On devient criminel d'affaires soit parce que l'on a fait des mauvaises affaires soit parce que les affaires étaient trop fructueuses. Les difficultés financières et le désir de maintenir à tout prix son entreprise, l'inexpérience ou l'incompétence qui entraînent parfois une sorte d'affolement du dirigeant devant les premières difficultés, l'idée qu'il est naturel, lorsque l'on a investi une partie de sa fortune dans une société et que viennent des moments délicats, de récupérer ses fonds pour les mettre à l'abri des créanciers sont autant de facteurs qui conduisent graduellement à la criminalité lorsque les affaires vont mal. Dans d'autres circonstances, une affaire trop

40. J. Pinatel (1970), note 13, p. 680. Pour une comparaison entre le criminel en col blanc et le criminel professionnel, voir Sutherland (1961), White Collar Crime, p. 217-233. 
florissante conduit, tout aussi insensiblement, les responsables à profiter illégalement des possibilités offertes par une économie d'abondance.

Quant aux facteurs internes de la criminalité, on a affirmé que le délinquant d'affaires, chez qui l'esprit d'entreprise s'allie au goût du jeu, appartiendrait à un type «colérique, facilement intolérant, optimiste et amoureux du changement $\gg$; il agit, nous dit-on, avec esprit de rivalité et d'agressivité ${ }^{11}$.

Mais il y a encore d'autres explications, car la victime a, elle aussi, un rôle à jouer d'ailleurs ambigu. En effet, dans certains cas, l'appât d'un gain très élevé a souvent tenté à l'origine ceux qui, par la suite, se disent victimes; dans d'autres cas, la victime n'attire pas la même compassion du public lorsqu'elle est constituée d'une masse anonyme (l'exemple est frappant en ce qui concerne les fraudes qui, à travers une administration finances, douanes, sécurité sociale, etc. - , affectent la population tout entière).

Finalement, il semble qu'aucune réprobation sociale véritable ne s'attache à la condamnation pénale de l'homme d'affaires, qui, dans les rares cas où il sera condamné, trouvera sans difficulté, dès sa libération, une nouvelle affaire à diriger, même à titre occulte lorsque jouent les interdictions professionnelles.

Certes, certains sondages d'opinion publique sur la fraude fiscale effectués récemment en France et en Belgique ont donné une appréciation nettement défavorable sur cette forme de criminalité ${ }^{42}$. Mais si l'on examine de plus près les motifs justifiant les réponses, on constate que la condamnation totale ou particulière de la fraude est essentiellement motivée par l'impossibilité de s'y livrer soi-même.

Comment expliquer cette indulgence à l'égard de la criminalité d'affaires ? Elle peut venir, soit d'une certaine solidarité du milieu, soit de la différence instinctive qu'établit le public entre

41. Voir note 39.

42. Voir Denuzière (1973), note 23, p. 101 , ainsi que le numéro spécial de la Revue de droit pénal et de criminologie, (n ${ }^{\circ \mathrm{s}} 2-3$, novembre-décembre 1973) intitulé * Atteintes au crédit. Fraude fiscale *, p. 183-213. En ce qui concerne les Etats-Unis, voir John P. et Robin S. Reed, e Refrains anciens et nouveaux sur le crime en col blanc ", Revue de science criminelle, no 4,1974, p. 783-806, qui offrent un aperçu de la littérature américaine relative à cet aspect de la criminalité d'affaires et présentent les résultats d'une enquête sur les attitudes de la population à l'égard de cette forme de criminalité. 
la criminalité violente et la criminalité astucieuse, soit le sentiment diffus que le délinquant d'affaires est un homme comme les autres mais qui n'a pas eu de chance. Au fond, c'est la vieille idée que n'est pas vraiment criminel celui qui n'est pas «dissemblable ».

\section{La réaction sociale}

La réaction sociale favorise, dans une certaine mesure, cette forme de délinquance. Le statut social des criminels d'affaires, nous venons de le voir, les met à l'abri d'un jugement méprisant, non seulement de leurs pairs, mais du public en général.

En principe, la société se doit d'agir contre la criminalité d'affaires; en pratique, sa tâche est loin d'être aisée, et des difficultés surgissent dès que l'on cherche à préciser quand et comment doit être menée son action.

En ce qui concerne le moment opportun où la société doit intervenir, on observe tout d'abord que la découverte des infractions est lente et difficile, le chiffre noir étant vraisemblablement très élevé dans ce domaine. Les dénonciations émanent plutôt de concurrents jaloux que des vérificateurs, inspecteurs ou commissaires aux comptes, qui sont néanmoins obligés à dénoncer; les victimes, indifférentes ou ignorantes le plus souvent, révèlent rarement ces infractions. Les premiers indices menant à une enquête proviennent généralement d'une décision du tribunal de commerce ou d'un tribunal administratif, d'une traite protestée ou d'un chèque impayé. Dans les pays européens, la qualification juridique des infractions pose des problèmes particulièrement compliqués (un même comportement est généralement susceptible de multiples qualifications), alors que dans les pays anglo-saxons le problème de la preuve rend difficile la condamnation. L'instruction de l'affaire comporte enfin des difficultés techniques qui exigent souvent la présence d'experts-comptables; par ailleurs, les moyens financiers et les relations dont disposent ces délinquants d'affaires leur permettent d'utiliser, avec une efficacité très grande, les possibilités légales, ce qui aboutit à ce que certaines affaires soient jugées définitivement bien après que les faits aient été commis, avec tous les inconvénients que ces longs délais comportent (disparition de preuves ou de témoins, oubli du scandale causé par l'affaire, etc.).

Quant aux modalités d'intervention, la nécessité de l'action répressive soulève immédiatement le problème des sanctions et 
de leur efficacité. D'abord le choix de la sanction. Les mesures pénales, rarement appliquées comme on vient de le voir, sont généralement prononcées par les tribunaux de juridiction criminelle ; la peine la plus redoutée semble être la prison ; l'amende - une dette de plus - n'a pas beaucoup de sens dans ce domaine; d'autres sanctions (incapacité d'exercer une profession, confiscation des biens, retrait du permis de conduire, publication et affichage de la condamnation) peuvent être efficaces à condition de bien les appliquer. Les sanctions administratives se caractérisent par l'absence de toute publicité dans leur application, ne présentent aucun caractère infamant ni intimidant, sont purement pécuniaires et leur application est confiée, en règle générale, à des fonctionnaires.

Afin de rendre plus efficace la lutte contre la criminalité d'affaires, certains pays ont eu recours à diverses mesures d'organisation. En République fédérale d'Allemagne, par exemple, on a institué, au Ministère public, ce qu'on appelle des "parquets lourds », au service desquels sont affectés des experts, des rapporteurs (généralement des commerçants diplômés) et des comptables. Ce sont, par ailleurs, des juges spécialisés qui occupent, depuis quelques années, les chambres de droit pénal économique, presque exclusivement consacrées au jugement de cette sorte de crimes. D'autres mesures consistent à inciter les autorités policières et judiciaires à échanger davantage les connaissances apprises de l'expérience et à centraliser l'observation obtenue ${ }^{43}$.

\section{CONCLUSION}

L'importance de la criminalité d'affaires et le peu de connaissances scientifiques que nous avons dans ce domaine rendent urgente la mise sur pied de recherches dans ce secteur de la délinquance.

Dans cette optique, un effort prioritaire devrait, à notre avis, être accordé aux points suivants :

A. La délimitation du champ de cette forme de criminalité. Que doit-on entendre par criminalité d'affaires? Quels critères retenir ? Doit-on se limiter à l'étude des crimes en col blanc, au sens où Sutherland les définissait, ou accepter un champ d'action plus large, qui engloberait toute la délinquance occupationnelle ? Quoi qu'il en soit, une analyse épidémiologique approfondie de

43. K. Tiedemann (1975). 
cette catégorie criminelle devient indispensable. Les difficultés seront grandes, en raison notamment de l'existence d'un chiffre noir particulièrement important de l'inaccessibilité de certaines sources d'information. Même les méthodes récentes d'investigation sur le chiffre noir (criminalité autoreportée, enquêtes de victimisation) semblent, au premier abord, peu efficaces en ce domaine. Le danger, dans ces conditions, est de s'évader de la stricte méthode scientifique et d'agir à la manière d'une sorte de journaliste supérieur.

B. L'étude clinique du délinquant d'affaires semble à la fois moins urgente et plus difficile (en raison du nombre restreint de personnes qui se font arrêter et condamner). Par contre, une approche socio-politique permettrait de mieux comprendre non seulement les motivations des criminels d'affaires mais également, et surtout, les mécanismes de passage à l'acte, notamment la tolérance, voire les éventuelles complicités, conscientes ou inconscientes, des hommes politiques et du législateur dans la commission de ce type de crimes.

C. La réaction sociale à la criminalité d'affaires. Celleci se situe soit sur un modèle formel (qui peut référer non seulement au système de contrôle social normalement compétent la justice pénale - mais aussi à d'autres systèmes de contrôle administrations, commissions, etc. - ) soit sur un mode informel. Il faudrait, dès lors, recenser tout d'abord les différents systèmes et sous-systèmes sociaux chargés de contrôler cette forme de déviance et analyser ensuite la façon dont ces systèmes réalisent cette fonction de contrôle social (transactions, mesures extra-judiciaires, sanctions pénales).

D. Enfin, le concept de « criminalité d'affaires $\gg$ a une grande importance en ce qui concerne les théories explicatives de la délinquance. Les théories criminologiques classiques, selon lesquelles le comportement délinquant est la conséquence plus ou moins directe de certaines pathologies personnelles et sociales, ont été élaborées à partir d'une idée fausse, ou du moins incomplète, du crime et du criminel. Ces théories ont, en effet, été construites sur la base d'échantillons pénitentiaires non représentatifs de la population criminelle globale; or, la criminalité d'affaires est une délinquance d'astuce, largement impunie, échappant en grande partie aux poursuites pénales et aux condamnations. 
L'explication que Sutherland donne de la criminalité en col blanc n'apporte pas une théorie générale, ni une structure explicative de la criminalité, encore qu'elle ait été étayée dès sa naissance par la double théorie des associations différentielles (au niveau individuel) et de l'organisation sociale différentielle (sur le plan de la société globale). C'est surtout sur la première de ces théories que des critiques sérieuses ont été formulées. Ainsi Clinard, étudiant le marché noir aux États-Unis au cours de la Deuxième Guerre mondiale, considérait que l'explication de Sutherland ne tenait pas compte des facteurs personnels, des pressions plus ou moins fortes exercées par les fournisseurs et les consommateurs, de l'inefficacité du gouvernement, insuffisamment outillé pour faire respecter une réglementation nouvelle aux États-Unis; dans ces conditions, il proposait d'ajouter aux hypothèses explicatives de Sutherland, le rôle possible de certains facteurs économiques, l'influence de plusieurs traits psychologiques des délinquants et, en particulier, des exécutifs des sociétés ${ }^{44}$.

Pour certains criminologues, le concept de criminalité d'affaires ne prend toute sa signification que si on le considère comme une application de la théorie générale de la stigmatisation. Dans la ligne de cette conception nouvelle de la conduite déviante, le traitement préférentiel accordé au criminel d'affaires s'expliquerait avant tout par la structure sociale et l'organisation de la réaction sociale ${ }^{45}$.

Tels sont les principaux points à approfondir en ce qui concerne l'étude scientifique de cette forme de criminalité, liée à la civilisation d'abondance et dont l'explication doit en somme être recherchée "dans le fossé qui se creuse entre la nature de l'homme et les formes de vie de la société contemporaine ${ }^{46}$.

44. Clinard (1952), note 17.

45. Kellens (1970), note 34 .

46. Pinatel (1970), note 13, p. 683. 